\title{
POWERS IN PRODUCTS OF TERMS OF PELL'S AND PELL-LUCAS SEQUENCES
}

\author{
JHON J. BRAVO, PRANABESH DAS, SERGIO GUZMÁN, AND SHANTA LAISHRAM
}

\begin{abstract}
In this paper, we consider the usual Pell and Pell-Lucas sequences. The Pell sequence $\left(u_{n}\right)_{n=0}^{\infty}$ is given by the recurrence $u_{n}=2 u_{n-1}+u_{n-2}$ with initial condition $u_{0}=0, u_{1}=1$ and its associated Pell-Lucas sequences $\left(v_{n}\right)_{n=0}^{\infty}$ is given by the recurrence $v_{n}=2 v_{n-1}+v_{n-2}$ with initial condition $v_{0}=2, v_{1}=2$.

Let $n, d, k, y, m$ be positive integers with $m \geq 2, y \geq 2$ and $\operatorname{gcd}(n, d)=1$. We prove that the only solutions of the Diophantine equation $u_{n} u_{n+d} \cdots u_{n+(k-1) d}=$ $y^{m}$ are given by $u_{7}=13^{2}$ and $u_{1} u_{7}=13^{2}$ and the equation $v_{n} v_{n+d} \cdots v_{n+(k-1) d}=$ $y^{m}$ has no solution. In fact we prove a more general result.
\end{abstract}

\section{INTRODUCTION}

There are a lot of integer sequences which are used in almost every field of modern sciences. For instance, the Fibonacci sequence is one of the most famous and curious numerical sequences in mathematics and has been widely studied in the literature. Also, there is the Pell sequence, which is as important as the Fibonacci sequence. The Pell sequence $\mathbf{u}:=\left(u_{n}\right)_{n=0}^{\infty}$ are defined by the recurrence $u_{n}=2 u_{n-1}+u_{n-2}$ for all $n \geq 2$ with $u_{0}=0$ and $u_{1}=1$ as initial conditions, and the Pell-Lucas sequence $\mathbf{v}:=\left(v_{n}\right)_{n=0}^{\infty}$ by the same recurrence relation but with initial conditions $v_{0}=2$ and $v_{1}=2$. Further details about Pell and Pell-Lucas sequences can be found, for instance, in $[2,6,7,8]$.

Explicit Binet formulas for $\mathbf{u}$ and $\mathbf{v}$ are well known. Namely, for all $n \geq 0$, we have that

$$
u_{n}=\frac{\alpha^{n}-\beta^{n}}{\alpha-\beta} \quad \text { and } \quad v_{n}=\alpha^{n}+\beta^{n},
$$

where $\alpha:=1+\sqrt{2}$ and $\beta:=1-\sqrt{2}$ are the roots of the characteristic equation $x^{2}-2 x-1=0$. Below we present the first few elements of the Pell and Pell-Lucas sequences:

$$
\begin{aligned}
& \mathbf{u}=\{0,1,2,5,12,29,70,169,408,985,2378,5741,13860,33461,80782, \ldots\} \\
& \mathbf{v}=\{2,2,6,14,34,82,198,478,1154,2786,6726,16238,39202,94642, \ldots\}
\end{aligned}
$$

2010 Mathematics Subject Classification: 11B37; 15A15; 11D61;

Keywords: Recurrence Sequences, Pell Sequences, Pell-Lucas Sequences, Arithmetic progressions. 
There are several papers in the literature dealing with Diophantine equations involving powers in products of consecutive integers, or in products of consecutive terms in arithmetic progressions. For example, Erdős and Selfridge [5] showed that a product of at least two consecutive integers is never a perfect power. For a survey, see [21]. On the other hand, Luca and Shorey in [9] addressed a similar question when the product of consecutive terms in arithmetic progressions is replaced by the product of terms in Lucas sequences whose indices form an arithmetic progression. In this paper, we consider Pell and Pell-Lucas sequences and solve it explicitly.

Let $n, d, k, b, y$ and $m$ be positive integers with $m \geq 2, \operatorname{gcd}(n, d)=1$ and $y>1$. For an integer $t$, we denote by $P(t)$ the largest prime divisor of $t$ with the convention $P( \pm 1)=1$. We put

$$
f(k, d)= \begin{cases}2 k & \text { if } d>1 \\ k & \text { if } d=1\end{cases}
$$

In this paper we consider the Diophantine equations

$$
u_{n} u_{n+d} \cdots u_{n+(k-1) d}=b y^{m}
$$

and

$$
v_{n} v_{n+d} \cdots v_{n+(k-1) d}=b y^{m}
$$

in positive integer unknowns $(n, d, k, b, y, m)$ with $P(b) \leq f(k, d)$. The special case when $k=b=1$ in (2) is due to Pethö [12] (see also Cohn [4]). Let us state their result since we shall use it later.

Theorem A. [Pethő [12], Cohn, [4]] The Diophantine equation $u_{n}=y^{m}$ in positive integers $n, y, m$ with $m \geq 2$ has only the solutions $(n, y, m)=(1,1, m)$ and $(7,13,2)$.

For a given $b$, it follows from results proved independently by Pethö [11] and Shorey and Stewart [19], that either one of equations (2) and (3) with $k=1$ or 2 implies that $n, d, y$ and $m$ are bounded by an effectively computable number depending only on the sequence and $b$. In fact, the preceding assertion with $b$ composed only of primes from a given finite set follows from the result of Pethő.

For $k \geq 3$, Luca and Shorey [9] proved that if $P(b) \leq f(k, d)$, then $k$ is bounded by an effectively computable number depending only on the sequence. It was also proved in [9] that if $P \geq 1$ is an integer such that $P(b) \leq P$, then equations (2) and (3) implies that $n, d, k, b, y$ and $m$ are bounded by an effectively computable number depending only on the sequence and $P$. Furthermore, Luca and Shorey in the same work showed that equation (2) with $u_{n}=F_{n}$ and $b=1$ has no solution except for $F_{1} F_{2}=1, F_{1} F_{6}=2^{3}$ and $F_{1} F_{12}=12^{2}$ where $F_{n}$ is the $n$th Fibonacci number. They proved it as an extension of a breakthrough result of Bugeaud, Mignotte and Siksek [3] which states that (2) with $u_{n}=F_{n}, k=1$ and $b=1$ implies $F_{1}=1, F_{2}=1, F_{6}=2^{3}$ and $F_{12}=12^{2}$. 
In this paper, we explicitly solve find all the solutions of (2) and (3) for the sequences $\mathbf{u}$ and $\mathbf{v}$. We prove the following results.

Theorem 1. Let $n, d, k, b, y, m$ be positive integers with $\operatorname{gcd}(n, d)=1, y>1$ and $m>1$. Then the equation

$$
u_{n} u_{n+d} \cdots u_{n+(k-1) d}=y^{m}
$$

implies

$$
u_{7}=13^{2} \text { and } u_{1} u_{7}=13^{2} .
$$

The equation

$$
v_{n} v_{n+d} \cdots v_{n+(k-1) d}=y^{m}
$$

has no solution.

In fact we prove stronger results.

Theorem 2. The only solutions of the Diophantine equation (2) are given by

$$
(n, d, k) \in\{(7, d, 1),(1,6,2),(2,5,2),(4,3,2),(1,3,3)\} .
$$

In fact

$$
u_{7}=13^{2}, u_{1} u_{7}=13^{2}, u_{2} u_{7}=2 \cdot 13^{2}, u_{4} u_{7}=2^{2} \cdot 3 \cdot 13^{2}, u_{1} u_{4} u_{7}=2^{2} \cdot 3 \cdot 13^{2} .
$$

Theorem 3. The only solutions of the Diophantine equation (3) are given by $k=$ $1, n=1, v_{1}=2 \cdot 1^{m}$ for any $m \geq 2$ and possibly $k=2, n=1, d>1, d \equiv 1(\bmod 4)$ and $v_{1+d}=2 \cdot 3^{b} y^{m}$ for some $b \geq 1$ and $m>2$. In particular, for $k \geq 3$, there is no solution.

We believe that $k=2, n=1, d>1, d \equiv 1(\bmod 4)$ and $v_{1+d}=2 \cdot 3^{b} y^{m}$ is not possible. It follows if the equation which we believe to be true. We state it as a conjecture.

Conjecture 4. The equation $2 x^{2}+1=3^{b} y^{m}$ has no solution in positive integers $x, b, y, m$ with $y>1, m>2$ and $b, m$ even.

We observe that Theorem 1 follows easily from Theorems 2 and 3 . We prove Theorem 2 in Section 4 and Theorem 3 in Section 5. For the proof of Theorem 3, we use the following result which is of independent interest.

Theorem 5. Let $n \geq 1, d>1$ and $k \geq 6$ with $\operatorname{gcd}(n, d)=1$. Then there is at least one $i, 0 \leq i<k$ with $P(n+i d)>k$ and $n+i d$ odd.

We prove Theorem 5 in Section 3. The preliminaries and lemmas for the proof of Theorems 2-5 are given in Section 2. 
This work was done during the visit of J. J. Bravo and S. Guzmán to Indian Statistical Institute, New Delhi under Indo-Mexican Joint Project. We thank DST, India and CONACyT, Mexico for their funding this joint project. J. J. Bravo was also partially supported by Universidad del Cauca, Colciencias from Colombia.

\section{Notations AND PRELiminaries}

For a real number $x>1$, let $\pi(x)$ denote the number of primes $p \leq x$. For a positive integer $\ell$, let $P(\ell)$ and $\omega(\ell)$ denote the greatest prime divisor and number of distinct prime divisors of $\ell$ and we put $P(1)=1$ and $\omega(1)=0$. We denote by $p_{1}=2, p_{2}=3, p_{3}=5, \ldots$ the sequence of prime numbers. We always write $p$ for a prime.

For a non-zero integer $\ell$ and a prime number $p$ we write $\operatorname{ord}_{p}(\ell)$ for the highest power of $p$ dividing $\ell$. For two positive integers $s$ and $t$ we write either $\operatorname{gcd}(s, t)$ or $(s, t)$ for the greatest common divisor of $s$ and $t$.

First we list some of the well-known properties for the sequences $\mathbf{u}$ and $\mathbf{v}$ which we will be using frequently.

Lemma 2.1. For the sequences $\mathbf{u}$ and $\mathbf{v}$, we have

(a) $u_{2 n}=u_{n} v_{n}$.

(b) $8 u_{n}^{2}-v_{n}^{2}=4(-1)^{n+1}$.

(c) $v_{2 n}=v_{n}^{2}-2(-1)^{n}$.

(d) $\operatorname{gcd}\left(u_{m}, u_{n}\right)=u_{(m, n)}$.

(e) If $m \mid n$ and $p$ is a prime dividing $\operatorname{gcd}\left(u_{m}, u_{n} / u_{m}\right)$, then $p$ divides $n / m$.

( $f)$ For $n \geq 3$, there exists a prime $p \mid u_{n}$ such that $p \nmid u_{m}$ for each positive integer $m<n$. Such a prime is called a primitive prime divisor of $u_{n}$ and is always congruent to \pm 1 modulo $n$.

(g) For $n \geq 2$, then exists a prime $p \mid v_{n}$ such that $p \nmid v_{m}$ for each positive integer $m<n$. Such a prime is called a primitive prime divisor of $v_{n}$ and is always congruent to \pm 1 modulo 2 .

As a consequence we have:

Corollary 2.2. Let $q$ be a odd prime and $r>0$ be any integer. Let $p$ be a prime.

(i) If $p \mid u_{q^{r}}$, then $p \geq 2 q-1$.

(ii) If $p \mid v_{q^{r}}$ and $p \neq 2$, then $p \geq 2 q-1$.

Proof. By Lemma $2.1(d), \operatorname{gcd}\left(u_{q^{r}}, u_{n}\right)=u_{1}=1$ if $q \nmid n$ and $\operatorname{gcd}\left(u_{q^{r}}, u_{q^{s}}\right)=u_{q^{s}}$ for every $1 \leq s<r$. Hence $p \mid u_{q^{r}}$ implies either $p$ is a primitive prime divisor of $u_{q^{r}}$ or a primitive prime divisor of $u_{q^{s}}$ for some $1 \leq s<r$. Hence $p \equiv \pm 1$ modulo $q^{u}$ for some 
$1 \leq u \leq r$ implying $p \equiv \pm 1$ modulo $2 q^{u}$ since $p \pm 1$ is even. Hence $p \geq 2 q^{u}-1 \geq 2 q-1$. This proves the assertion $(i)$.

For any $r \geq 1$ and $t \geq 1$, observe that

$$
\operatorname{gcd}\left(v_{q^{r}}, v_{t}\right) \mid \operatorname{gcd}\left(u_{2 q^{r}}, u_{2 t}\right)=u_{2 \operatorname{gcd}\left(q^{r}, t\right)}=u_{2} \text { or } u_{2 q^{s}}
$$

according as $q \nmid t$ or $q \mid t, s=\min \left(r, \operatorname{ord}_{q}(t)\right)$.

Let $p \mid v_{q_{r}}, p \neq 2$. If $p$ is a primitive prime divisor of $v_{q^{s}}$ for any $1 \leq s \leq r$, then by Lemma $2.1(g), p \geq 2 q^{s}-1 \geq 2 q-1$. Hence assume that $p$ is not a primitive prime divisor of $v_{q^{s}}$ for any $1 \leq s \leq r$. Then $p \mid v_{t}$ for some $1<t<q^{r}$. Since $p \neq 2$, we have $p \mid u_{2 q^{s}}$ where $1 \leq s=\operatorname{ord}_{q}(t)<r$. Let $t$ be the one with minimal $s$. Observe that $\operatorname{gcd}\left(v_{q^{r}}, u_{q^{s}}\right)=1$ since $\operatorname{gcd}\left(v_{q^{r}}, u_{q^{r}}\right)=1$ and $u_{q^{s}} \mid u_{q^{r}}$. Thus $p \mid \frac{u_{2 q^{s}}}{u_{q^{s}}}=v_{q^{s}}$. Since $p$ is not a primitive divisor of $v_{q^{s}}, p \mid v_{t}$ for some $1<t<q^{s}$ implying $p \mid u_{2 q^{s}{ }_{1}}$ where $1 \leq s_{1}=\operatorname{ord}_{q}(t)<s$ contradicting the minimality of $s$ with $p \mid u_{2 q^{s}}$. Hence assertion (ii) follows.

In the next lemma, we derive some algebraic properties for the sequences $\mathbf{u}$ and $\mathbf{v}$.

Lemma 2.3. The following properties for $\mathbf{u}$ and $\mathbf{v}$ hold:

(a) For all $n \in \mathbb{N}$, we have that $\operatorname{ord}_{2}\left(u_{n}\right)=\operatorname{ord}_{2}(n)$ and $\operatorname{ord}_{2}\left(v_{n}\right)=1$.

(b) $3 \mid v_{n}$ if and only if $n \equiv 2(\bmod 4)$.

(c) $5 \nmid v_{n}$ for any $n$.

(d) For $(n, d)=1$, we have $\operatorname{gcd}\left(v_{n}, v_{n+d}\right)=\operatorname{gcd}\left(v_{n}, v_{n+2 d}\right)=2 \operatorname{and} \operatorname{gcd}\left(v_{n}, v_{n+3 d}\right) \mid 14$.

(e) Let $m \mid n$ and $\frac{n}{m}$ is odd. If a prime $p \mid \operatorname{gcd}\left(v_{m}, \frac{v_{n}}{v_{m}}\right)$, then $p \mid \frac{n}{m}$.

Proof. (a) From Lemma $2.1(b)$, we get $4 \mid v_{n}^{2}$ but $8 \nmid v_{n}^{2} \operatorname{implying} \operatorname{ord}_{2}\left(v_{n}\right)=1$. For $u_{n}$ 's, we note from the recurrence for $\mathbf{u}$ that $u_{n} \equiv u_{n-2}(\bmod 2)$ for all $n \geq 2$. Using this and $u_{1}=1$, we obtain $u_{n}$ is odd when $n$ is odd. Let $n$ be even and write $n=2^{e} t$ with $e$ and $t$ are positive integers and $t$ is odd. Repeatedly applying Lemma $2.1(a)$, we have

$$
u_{n}=u_{t}\left(\prod_{i=0}^{e-1} v_{2^{i} t}\right) .
$$

Since $t$ is odd, $u_{t}$ is odd. This together with the fact that $\operatorname{ord}_{2}\left(v_{2^{i} t}\right)=1$ for each $0 \leq i<e$ implies $\operatorname{ord}_{2}\left(u_{n}\right)=e=\operatorname{ord}_{2}(n)$, which proves $(a)$.

The assertions $(b)$ and $(c)$ follow easily by considering the sequence $\mathbf{v}$ modulo 3 and 5, respectively. For the proof of $(d)$, we observe from Lemma $2.1(a)$ and $(d)$ that

$$
g_{r}:=\operatorname{gcd}\left(v_{n}, v_{n+r d}\right) \mid \operatorname{gcd}\left(u_{2 n}, u_{2(n+r d)}\right)=u_{2 \cdot \operatorname{gcd}(n, n+r d)}=u_{2 \cdot \operatorname{gcd}(n, r)}
$$


for any $r>0$. Also by $(a), 2|| g_{r}$. Hence for $r=1$, we get $g_{1}=2$. For $r=2, g_{2} \mid u_{4}=12$. If $3 \mid g_{2}$, then from $(b), n \equiv 2(\bmod 4)$ and $n+2 d \equiv 2(\bmod 4)$ which is not possible. Hence $g_{2}=2$. The assertion $(d)$ now follows from $(c)$ and the fact that $g_{3} \mid u_{6}=70$.

Finally we prove $(e)$. We observe that $\alpha^{m} \equiv-\beta^{m}(\bmod p)$ since $p \mid v_{m}$. If $n=m \ell$ with $\ell$ odd, we have

$$
\begin{aligned}
\frac{v_{n}}{v_{m}}=\frac{\left(\alpha^{m}\right)^{\ell}+\left(\beta^{m}\right)^{\ell}}{\alpha^{m}+\beta^{m}} & =\left(\alpha^{m}\right)^{\ell-1}-\left(\alpha^{m}\right)^{\ell-2}\left(\beta^{m}\right)+\cdots+\left(\beta^{m}\right)^{\ell-1} \\
& \equiv \ell \alpha^{m(\ell-1)} \equiv \ell \beta^{m(\ell-1)} \quad(\bmod p) .
\end{aligned}
$$

Consequently $p^{2} \mid \ell^{2}(\alpha \beta)^{m(\ell-1)}$ implying $p \mid \ell$ as desired.

The following result follows from Lemma $2.3(e)$.

Corollary 2.4. Suppose that $m \mid n$ and $\frac{n}{m}$ is odd. If $P\left(\frac{n}{m}\right)<p$ for any odd prime $p$ dividing $v_{m}$, then $\operatorname{gcd}\left(v_{m}, \frac{v_{n}}{v_{m}}\right)=1$.

Proof. Let $m \mid n, \frac{n}{m}$ is odd and $d=\operatorname{gcd}\left(v_{m}, v_{n} / v_{m}\right)$. Suppose $d>1$. Then there is a prime $p$ such that $p \mid v_{m}$ and $p \mid \frac{v_{n}}{v_{m}}$. By Lemma $2.3(e)$, we get $p \mid \frac{n}{m}$ since $\frac{n}{m}$ is odd. This implies that $p$ is an odd prime with $p \leq P\left(\frac{n}{m}\right)$. This contradicts the assumption of the Corollary. Thus $d=1$.

The next two lemmas give all solutions of (3) and (2) when $k=1$.

Lemma 2.5. Let $n, y, m \in \mathbb{N}$ with $m \geq 2$. Then the equation $v_{n}=y^{m}$ has no integer solutions and the equation $v_{n}=2 y^{m}$ has the only integer solution $v_{1}=2 \cdot 1^{m}$.

Proof. By Lemma $2.3(a)$, we have $\operatorname{ord}_{2}\left(v_{n}\right)=2$ implying that $v_{n}=y^{m}$ has no integer solutions. For solving the equation $v_{n}=2 y^{m}$, we use Lemma $2.1(b)$. When $n$ is odd we obtain

$$
2 u_{n}^{2}-y^{2 m}=1
$$

By $\left[1\right.$, Theorem 1.1], we see that the only solution is given by $u_{n}=1, y=1$ implying then $n=1$ and $v_{1}=2 \cdot 1^{m}$. When $n$ is even, we have

$$
2 u_{n}^{2}=y^{2 m}-1=\left(y^{m}-1\right)\left(y^{m}+1\right) .
$$

Since $\operatorname{gcd}\left(y^{m}+1, y^{m}-1\right)=2$, we get either $y^{m}-1=2 x_{1}^{2}, y^{m}+1=x_{2}^{2}$ or $y^{m}-1=$ $x_{2}^{2}, y^{m}+1=2 x_{1}^{2}$ where $x_{1}$ is odd and $x_{2}$ is even. Catalan's conjecture, now Mihailescu's Theorem implies that $y^{m}+1=x_{2}^{2}$ or $y^{m}-1=x_{2}^{2}$ has no integer solutions when $x_{2}$ is even. Hence the result.

Lemma 2.6. The only solutions of the Diophantine equation

$$
u_{n}=2^{\alpha} 3^{\beta} z^{m}
$$


in positive integers $n, z, m$ with $m \geq 2$ and non-negative integers $\alpha, \beta$ are given by $n=1,2,4,7$.

Proof. If $\alpha=\beta=0$, then by Theorem $A$, we find that the solutions of (5) are given by $n=1$ and $n=7$. Hence, from now on we assume that $\alpha+\beta>0$. By Lemma 2.1 (a) and $(b)$, we get $n$ is even. Let $n=2^{e} t$, where $e$ and $t$ are positive integers with $t$ odd. Since $\operatorname{ord}_{2}\left(u_{n}\right)=\operatorname{ord}_{2}(n)$, it follows that $e=\alpha$ and (5) can be rewritten as

$$
u_{t}\left(\prod_{i=0}^{e-1} v_{2^{i} t}\right)=2^{e} 3^{\beta} z^{m} .
$$

Note that if $i<j$, then

$$
\operatorname{gcd}\left(v_{2^{i} t}, v_{2^{j} t}\right)\left|\operatorname{gcd}\left(u_{2^{i+1} t}, v_{2^{j} t}\right)\right| \cdots \mid \operatorname{gcd}\left(u_{2^{j} t}, v_{2^{j} t}\right)=2 .
$$

Thus, $\operatorname{gcd}\left(v_{2^{i} t}, v_{2^{j} t}\right)=2$ because $v_{2^{i} t}$ and $v_{2^{j} t}$ are both even numbers. In addition, since $t$ is odd, we get $\operatorname{gcd}\left(u_{t}, v_{2^{i} t}\right)=1$ for all $i$. Hence it follows from (6) that $u_{t}=z_{1}^{m}$ for some $z_{1} \mid z$ and there is some positive integer $i_{0}$ in the interval $[0, e-1]$ for which $v_{2^{i} 0 t}=2 \cdot 3^{\beta} z_{2}^{m}$ for some integer $z_{2} \mid z$. Then $t \in\{1,7\}$ by Theorem $A$. If $e=1$, then $n \in\{2,14\}$ and $n=14$ is not a solution for (5) and $n=2$ is already listed. Thus $e>1$. Then for every $0 \leq i \leq e-1, i \neq i_{0}$, we get $v_{2^{i} t}=2 \cdot z^{\prime m}$ for some $z^{\prime} \mid z$ implying $2^{i} t=1$ by Lemma 2.5. Thus $t=1$ and $e-2 \leq 0$ or $n \in\{1,2,4\}$. Hence the assertion.

We also need the following lemma which follows from [23, Theorem 6.3].

Lemma 2.7. The solutions of Diophantine equations
(1) $3^{a}+5^{b}=2 \cdot 7^{c}$
(2) $3^{a}+7^{c}=2 \cdot 5^{c}$
(4) $1+3^{a}=2 \cdot 5^{b} 7^{c}$;
(5) $1+5^{b}=2 \cdot 3^{a} 7^{c}$
(3) $5^{b}+7^{c}=2 \cdot 3^{a}$
(7) $1+3^{a} 5^{b}=2 \cdot 7^{c}$
(8) $1+3^{a} 7^{c}=2 \cdot 5^{a}$;
(6) $1+7^{c}=2 \cdot 3^{a} 5^{b}$;
(9) $1+5^{b} 7^{c}=2 \cdot 3^{a}$

in non negative integers $a, b, c$ with $\max \left(3^{a}, 5^{b}, 7^{c}\right)>1$ are given by

$$
\begin{array}{llll}
3^{2}+5^{1}=2 \cdot 7^{1} ; & 3^{1}+7^{1}=2 \cdot 5^{1} ; & 3^{5}+7^{1}=2 \cdot 5^{3} ; & 1+3^{2}=2 \cdot 5^{1} 7^{0} \\
1+5^{1}=2 \cdot 3^{1} 7^{0} ; & 1+5^{3}=2 \cdot 3^{2} 7^{1} ; & 1+7^{2}=2 \cdot 3^{0} 5^{2} ; & 1+5^{1} 7^{0}=2 \cdot 3^{1}
\end{array}
$$

Proof. We observe that the equations (1) - (9) are special cases of the the equation $x+y=z$ with $P(x y z) \leq 13$ and $\operatorname{gcd}(x, y)=1$. In [23, Theorem 6.3], it was shown that such equations $x+y=z$ with $x<y$ has exactly 545 solutions. Of them, 514 satisfy

$$
\begin{aligned}
& \operatorname{ord}_{2}(x y z) \leq 12, \quad \operatorname{ord}_{3}(x y z) \leq 7, \quad \operatorname{ord}_{5}(x y z) \leq 5, \\
& \operatorname{ord}_{7}(x y z) \leq 4, \quad \operatorname{ord}_{11}(x y z) \leq 3, \quad \operatorname{ord}_{13}(x y z) \leq 4
\end{aligned}
$$


and remaining 31 solutions were listed. We checked that none of the listed 31 solutions give any solution for the equations (1) - (9). Further we check for solutions with $a \leq 7, b \leq 5$ and $c \leq 4$ and find that only solutions are those listed in the assertion of the lemma.

Lemma 2.8. Let $x>10$ be an integer. Then the interval $(2 x / 3, x]$ contains a prime.

Proof. It suffices to prove $\pi(x)-\pi(2 x / 3) \geq 1$ holds for all $x>10$. From the inequalities

$$
\frac{x}{\log x-0.5}<\pi(x)<\frac{x}{\log x-1.5}, \quad x>67
$$

(see [13]), it follows that it is enough to check the inequality

$$
\frac{x}{\log x-0.5}-\frac{(2 x / 3)}{\log (2 x / 3)-1.5} \geq 1 \text {. }
$$

But the last inequality holds for all $x \geq 150$. This implies $\pi(x)-\pi(2 x / 3) \geq 1$ whenever $x \geq 150$. For the values $x \in[10,149]$, we check using exact values that the assertion of the Lemma is valid.

We also need some results on the greatest prime factor of a product of consecutive terms of an arithmetic progression. The following result is due to Sylvester [22] for $d=1$ and Shorey and Tijdeman [20] for $d>1$.

Lemma 2.9. Let $k \geq 3, n \geq 1, d \geq 1$ with $\operatorname{gcd}(n, d)=1$. Then

$$
P\left(\prod_{i=0}^{k-1}(n+i d)\right)>k \text { for } \begin{cases}n>k & \text { if } d=1 \\ n \geq 1 & \text { if } d>1 \text { unless }(n, d, k)=(2,7,3) .\end{cases}
$$

We also need the following sharpenings which are contained in Laishram and Shorey [15, Theorem 1] and [16, Theorem 1].

Lemma 2.10. Let $k \geq 4, n \geq 1, d \geq 1$ with $\operatorname{gcd}(n, d)=1$. Then

$$
P\left(\prod_{i=0}^{k-1}(n+i d)\right)>2 k \text { for } \begin{cases}n>\max \left(k+13, \frac{279 k}{262}\right) & \text { if } d=1 \\ n \geq 1 & \text { if } d>2\end{cases}
$$

unless $k=4,(n, d) \in\{(1,3),(1,13),(3,11)\}$ and $k=10,(n, d)=(1,3)$.

For $d=2$, we have the following result which is contained in [17, Theorem 3].

Lemma 2.11. Let $k \geq 2$ and $n$ odd with $n>2 k$. Then

$$
P\left(\prod_{i=0}^{k-1}(n+2 i)\right)>3.5 k
$$

unless $(n, k) \in\{(5,2),(7,2),(25,2),(243,2),(9,4),(13,5),(17,6),(15,7),(21,8),(19,9)\}$. 
We also need the following result which follows from Saradha, Shorey and Tijdeman [18] for $k \in\{6,7,8\}$ and Laishram and Shorey [14, Theorem 1] for $k \geq 9$.

Lemma 2.12. Let $k \geq 6, n \geq 1, d>2$ with $d$ even and $\operatorname{gcd}(n, d)=1$. Then

$$
\omega\left(\prod_{i=0}^{k-1}(n+i d)\right) \geq \pi(2 k) \quad \text { unless }(n, d, k)=(1,4,7) .
$$

\section{Proof of TheOREM 5}

For the proof of Theorem 5, we need the following result which is a refinement of Laishram and Shorey [16, Theorem 1].

Lemma 3.1. Let $n \geq 1, d>2$ be even which is not a power of 2 and $k \geq 3$ with $\operatorname{gcd}(n, d)=1$. Then

$$
P\left(\prod_{i=0}^{k-1}(n+i d)\right)>2 k+1
$$

unless $k=3,(n, d) \in\{(1,124),(7,118)\}$.

Proof. Let $n \geq 1, d>2$ be even is not a power of 2 and $k \geq 4$ with $\operatorname{gcd}(n, d)=1$. Clearly $(11)$ holds when $(n, d, k)=(1,4,7)$. Hence we take $(n, d, k) \neq(1,4,7)$. Assume that $P\left(\prod_{i=0}^{k-1}(n+i d)\right) \leq 2 k+1$.

Let $k=3$. Then $P(n(n+d)(n+2 d)) \leq 7$. Since $d$ is even, $n$ is odd and hence $\operatorname{gcd}(n+$ $i d, n+j d)=1$ for $0 \leq i<j \leq 2$. If $n>1$, we get $\{n, n+d, n+2 d\}=\left\{3^{a}, 5^{b}, 7^{c}\right\}$. If $n=1$, then $(n+d)(n+2 d)=(1+d)(1+2 d)=3^{a} 5^{b} 7^{c}$. Using $n+(n+2 d)=2(n+d)$, we get one of equations (1) - (9) listed in Lemma 2.7. We obtain from the solutions listed in Lemma 2.7 that $d=2, n \in\{1,3,5\}$ or $(n, d) \in\{(1,4),(1,24),(1,124),(7,118)\}$. Since $d>2$ is not a power of 2 and $3 \nmid d$, we have $(n, d) \in\{(1,124),(7,118)\}$ which is listed in assertion of the lemma.

Thus $k \geq 4$. By $(8)$, we have $P\left(\prod_{i=0}^{k-1}(n+i d)\right)=2 k+1$ implying $2 k+1$ is a prime. Hence $k \geq 5$. Further $\omega\left(\prod_{i=0}^{k-1}(n+i d)\right) \leq \pi(2 k+1)-1=\pi(2 k)$ since $n+i d$ are all odd for $0 \leq i<k$. This together with (10) implies $\omega\left(\prod_{i=0}^{k-1}(n+i d)\right)=\pi(2 k)$ when $k \geq 6$. Further for $k \geq 6$, if $p \mid d$ for some $3 \leq p \leq 2 k+1$, then $P\left(\prod_{i=0}^{k-1}(n+i d)\right) \geq$ $p_{\pi(2 k)+2}>2 k+1$ since $d$ is also even. Thus $p \mid d$ with $p \geq 3$ implies $p \geq 2 k+3$ when $k \geq 6$. When $k=5$, if $p \mid d$ for some $3 \leq p \leq 11$, then after deleting terms divisible by primes $3 \leq p \leq 11$, we are left with two terms $n+i_{0} d, n+i_{1} d, 0 \leq i_{0}<i_{1}$ such that $\left(n+i_{0} d\right)\left(n+i_{1} d\right) \mid 3$ which is not possible since $d>2$ is even. Hence for $k \geq 5$, we have $p \mid d$ with $p \geq 3$ implies $p \geq 2 k+3$. In particular, $d \geq 2(2 k+3)$ since $d$ is even. 
Let $k \in\{5,6\}$. After deleting terms divisible by primes $7 \leq q \leq 2 k+1$, we are left with three distinct terms $n+i_{0} d, n+i_{3} d, n+i_{5} d$ such that $p \mid\left(n+i_{0} d\right)\left(n+i_{3} d\right)\left(n+i_{5} d\right)$ imply $p \in\{3,5\}$ and 3 divides to a maximal power in $n+i_{3} d$ and 5 divides to a maximal power in $n+i_{5} d$. Hence $\left(n+i_{0} d\right) \mid 15$. If $i_{0}>0$, then $n+d \leq n+i_{0} d \leq 15$ contradicting $d \geq 2(2 k+3)$. Thus $i_{0}=0$ and $n=n+i_{0} d \in\{1,3,5,15\}$. Then we have the following possibilities:

$$
\begin{array}{ll}
n=1: & n+i_{3} d=3^{a}, n+i_{5} d=3^{\delta} 5^{b} \text { where } i_{3} \neq 3, i_{5} \neq 5 \\
n=3: & n+3 d=3^{a}, n+i_{5} d=5^{b} \quad \text { where } i_{3}=3, i_{5} \neq 5 \\
n=5: & n+i_{3} d=3^{a}, n+5 d=3^{\delta} 5^{b} \text { where } i_{3} \neq 3, i_{5}=5 \\
n=15: & n+3 d=3^{a}, n+5 d=5^{b} \quad \text { where } i_{3}=3, i_{5}=5
\end{array}
$$

and $a>0, b>0$ are integers, $3^{\delta} \in\{1,3\}$ and $3^{\delta}=3$ only if $i_{5}-i_{3}= \pm 3$. From the equality

$$
i_{5}\left(n+i_{3} d\right)-i_{3}\left(n+i_{5} d\right)=\left(i_{5}-i_{3}\right) n,
$$

we obtain an equation of the form $3^{r}-2^{\gamma} 5^{s}= \pm 1$ or $2^{\gamma} 3^{r}-5^{s}= \pm 1$ or $3^{r}-5^{s}=$ $\pm 2^{\gamma}$ where $2^{\gamma} \in\{1,2,4\}$. Observe that for the above terms, the pairwise ged $g \in$ $\{1,2,3,5,6,15\}$ and further $\left(n+i_{3} d\right) \mid \frac{3^{r} g}{i_{5}}$ and $\left(n+i_{5} d\right) \mid \frac{5^{s} g}{i_{3}}$. Special cases Catalan's Conjecture and the results of Nagell [10](see [16, Lemmas 2, 3]) along with [16, Lemma $4]$ imply that the only solutions are given by

$$
3-5=-2,3^{2}-5=4,3^{3}-5^{2}=2,3^{2}-2 \cdot 5=-1,2 \cdot 3-5=1 .
$$

Bringing back to the original equation and from $d \geq 2(2 k+3)$, we find that no such $n$ and $d$ exists.

Hence $k \geq 7$ and since $2 k+1$ is a prime, we have $k \geq 8$. Let $k \in\{8,9,11\}$. After deleting terms divisible by primes $11 \leq p \leq 2 k+1$, we are left with 5 or 6 terms terms divisible by $3,5,7$ according as $k \in\{8,9\}$ or $k=11$, respectively. If $7 \mid n$ or 7 divides exactly one of $n+i d$, we are left with a term $n+i_{0} d, i_{0}>0$ such that $\left(n+i_{0} d\right) \mid 15$ if $k \in\{8,9\}$ or $\left(n+i_{0} d\right) \mid 45$ if $k=11$. Since $d \geq 2(2 k+3)$, this is not possible. This is the case when $k=8$. When $k=11$, if 7 divides exactly two terms different from $n$, we are still left with a term $n+i_{0} d, i_{0}>0$ such that $\left(n+i_{0} d\right) \mid 45$ and this is not possible. Thus $k=9$ and we can assume that $7|(n+d), 7|(n+8 d)$ and $P((n+d)(n+8 d)) \leq 7$. After removing the terms divisible by $3,5,7$ to the maximal power, we are left with with a term $\left(n+i_{0} d\right) \mid 15$. If $i_{0}>0$, we a get a contradiction and hence $i_{0}=0, n \in\{1,3,5,15\}$. Further we have either $(n+d) \mid 105$ or $(n+8 d) \mid 105$ which together with $d \geq 2 \cdot 21$ implies that $(n+d) \mid 105$. This forces $n=1$ since $\operatorname{gcd}(d, 3 \cdot 5)=1$ and we have $(1+d) \mid 105$. Since $p \mid d$ implies $p>2 k+1=19$, no such $d$ exists. Hence the assertion (11) is valid.

Thus $k \geq 12$ implying $k \geq 14$ since $2 k+1$ is a prime. We follow the proof as in [14, Section 3] by taking $R=\pi(2 k)-\pi_{d}(k)$ where $\pi_{d}(k)$ is the number of primes $\leq k$ 
which are coprime to $d$. We have $\pi_{d}(k)=\pi(k)-1$ since $d$ is even and $p \mid d$ with $p \geq 3$ implies $p>2 k+1$. We obtain

$$
d^{k-\pi(2 k)-1} \leq(k-2) \cdots(k-\pi(2 k)) 2^{-\operatorname{ord}_{2}(k-2) !}
$$

and

$$
d \leq \exp \left[\frac{\frac{2 \log (k-2)}{\log 2 k}\left(1+\frac{1.2762}{\log 2 k}\right)-\left(1-\frac{3}{k}\right) \log 2}{1-\frac{2}{\log 2 k}\left(1+\frac{1.2762}{\log 2 k}\right)-\frac{1}{k}}\right] .
$$

We observe that the right hand side of (13) is a decreasing function of $k$. We find that $d<50$ at $k=40$ and hence $d<50$ for all $k \geq 40$. Since $d>2(k+1)$, we get a contradiction for $k \geq 40$. Hence $k<40$. We get from (12) that $d \leq 22$ for $14 \leq k<40, k \neq 16$. This is a contradiction since $d \geq 2(2 k+3)$ and $2 k+1$ is a prime.

Proof of Theorem 5: Let $d$ be even. Then $n$ is odd and each of $n, n+d, \ldots, n+$ $(k-1) d$ is odd. By Lemma 2.9, the assertion is valid. Thus we now assume $d$ is odd. Then the odd terms of among $\{n, n+d, \cdots, n+(k-1) d\}$ are given by

$$
\begin{array}{r}
n, n+2 d, \ldots, n+2\left[\frac{k-1}{2}\right] d \text { if } n \text { is odd } \\
n+d, n+3 d, \ldots, n+d+2\left[\frac{k-2}{2}\right] d \text { if } n \text { is even }
\end{array}
$$

and hence there are at least $1+\left[\frac{k-2}{2}\right]=\left[\frac{k}{2}\right]$ consecutive odd terms. Since $k \geq 6$, $\left[\frac{k}{2}\right] \geq 3$. By Lemma 3.1, the greatest prime factor of these consecutive odd terms is at least $2\left[\frac{k}{2}\right]+3 \geq 2\left(\frac{k-1}{2}\right)+3>k$ except possibly when $k \in\{6,7\}$ and $(n, 2 d) \in$ $\{(1,124),(7,118)\}$ if $n$ is odd and $(n+d, 2 d) \in\{(1,124),(7,118)\}$ if $n$ is even. Since $d$ is odd and the assertion for Theorem 5 is valid when $k=7,(n, 2 d)=(7,118)$, all this possibilities are excluded. This completes the proof of Theorem 5 .

\section{Proof of Theorem 2}

We need the following main lemma for the proof of Theorem 2.

Lemma 4.1. Suppose there is an $i, 0 \leq i<k$ with $Q:=P(n+i d)>\frac{1+f(k, d)}{2}, Q \neq 7$ and $Q \nmid(n+j d)$ for $0 \leq j<k, j \neq i$. Then the equation (2) has no solution in positive integers $(n, d, k, b, y, m)$ with $P(b) \leq f(k, d)$.

Proof. Suppose the conditions of Lemma 4.1 are satisfied. Let $0 \leq i<k$ be such that $Q:=P(n+i d)>\frac{1+f(k, d)}{2}$. Then $2 Q-1>f(k, d)$. Further write $n+i d=Q_{1} t$ where $Q_{1}=Q^{\nu}, \nu=\operatorname{ord}_{Q}(n+i d)$ and $\operatorname{gcd}(Q, t)=1$. Then $P(t)<Q$. From $(2)$, we get

$$
u_{Q_{1}} \cdot \frac{u_{n+i d}}{u_{Q^{\nu}}} \prod_{j \neq i} u_{n+j d}=b y^{m} .
$$


By Corollary $2.2(i)$, we have $p \geq 2 Q-1$ for every prime $p \mid u_{Q_{1}}$.

We now argue exactly as in [9]. If $p$ is a prime such that $p \mid \operatorname{gcd}\left(u_{Q_{1}}, u_{n+i d} / u_{Q_{1}}\right)$, then it is well known that $p \mid t$ and hence $p \leq P(t)<Q$. This contradicts $p \geq 2 Q-1$ since $p \mid u_{Q_{1}}$. Hence $\operatorname{gcd}\left(u_{Q_{1}}, u_{n+i d} / u_{Q_{1}}\right)=1$.

We now look at $\operatorname{gcd}\left(u_{Q_{1}}, u_{n+j d}\right)$ for $j \neq i$. Since $P(n+j d)<Q$ for $j \neq i$, we have $\operatorname{gcd}\left(Q_{1}, n+j d\right)=1$ for $j \neq i$ and hence

$$
\operatorname{gcd}\left(u_{Q_{1}}, u_{n+j d}\right)=u_{\left(Q_{1}, n+j d\right)}=1 .
$$

Since $p \geq 2 Q-1>f(k, d)$ for every $p \mid u_{Q_{1}}$ and $P(b) \leq f(k, d)$, it follows from (14) that $u_{Q_{1}}$ is a perfect $m$-th power. By Theorem $\mathrm{A}$ and $Q>1$, we get $Q_{1}=Q=$ $7, m=2$ and $u_{Q_{1}}=13^{2}$. Since $Q \neq 7$, this is a contradiction. Hence the assertion.

Proof of Theorem 2: By Lemma 2.6 we may assume that $k \geq 2$. Let $k=2$. Then equation (2) becomes

$$
u_{n} u_{n+d}=b y^{2} \quad \text { with } \quad P(b) \leq 3 .
$$

Since $\operatorname{gcd}\left(u_{n}, u_{n+d}\right)=1$, we obtain $u_{n+d}=2^{\alpha} 3^{\beta} y_{1}^{2}$ for some integer $y_{1} \geq 1$ and non-negative integers $\alpha, \beta$ and further $\beta=0$ when $d=1$. By Lemma 2.6, we get $n+d \in\{1,2,4,7\}$. For these possibilities of $n$ and $d$, we check that only solutions of $u_{n} u_{n+d}=b y^{2}$ with $P(b) \leq 3$ are given by $(n, d) \in\{(1,6),(2,5),(4,3)\}$.

Let $k=3$. We need to solve

$$
u_{n} u_{n+d} u_{n+2 d}=b y^{2} \quad \text { with } \quad P(b) \leq 5 .
$$

Observe that $\operatorname{gcd}\left(u_{n+i d}, u_{n+(i+1) d}\right)=1$ for $i=0,1$ and further

$$
\operatorname{gcd}\left(u_{n}, u_{n+2 d}\right)=u_{\operatorname{gcd}(n, n+2 d)} \mid u_{2}=2 .
$$

If $P(b)<5$, then $u_{n} u_{n+d}=b_{1} y_{1}^{2}$ and $u_{n+d} u_{n+2 d}=b_{2} y_{2}^{2}$ for some integers $b_{1}, b_{2}, y_{1}, y_{2}$ with $P\left(b_{1} b_{2}\right) \leq 3$. By case $k=2$, we get $(n, d) \in\{(1,6),(2,5),(4,3)\}$ and also $(n+d, d) \in\{(1,6),(2,5),(4,3)\}$. This is not possible. Hence $P(b)=5$. Further 5 divides at most one of $u_{n}, u_{n+d}$ or $u_{n+2 d}$. Therefore either $u_{n} u_{n+d}=b_{1} y_{1}^{2}$ or $u_{n+d} u_{n+2 d}=b_{2} y_{2}^{2}$ or $u_{n} u_{n+2 d}=b_{3} y_{3}^{2}$ with $P\left(b_{1} b_{2} b_{3}\right) \leq 3$. Then the case $k=2$ gives either $(n, d) \in\{(1,6),(2,5),(4,3)\}$ or $(n+d, d) \in\{(1,6),(2,5),(4,3)\}$ or $(n, 2 d) \in\{(1,6),(2,5),(4,3)\}$. These possibilities gives the only solution $n=1, d=3$ of $(15)$ or $u_{1} u_{4} u_{7}=2^{3} \cdot 3 \cdot 13^{2}$.

Thus $k \geq 4$. Suppose $d>1$ or $d=1, n>k$. We observe that a prime $>k$ divide at most term $n+i d$ with $i \in[0, k)$. Hence by Lemmas 4.1 and 2.9, we have $k \in\{4,5,6\}$. Further $P\left(\prod_{i=0}^{k-1}(n+i d)\right)=7$ and $5 \nmid \prod_{i=0}^{3}(n+i d)$ if $k=4$. For $d=1, k<n \leq k+13$ or $d=2, n \leq 2 k$ or $(n, d, k) \in\{(1,3,4),(1,13,4),(3,11,4),(9,2,4),(13,2,5),(17,2,6)\}$. we check that this is not possible except when $(n, d, k)=(6,1,4)$. For $(n, d, k)$ 
different from these, we obtain from Lemmas 2.10 and 2.11 that $P\left(\prod_{i=0}^{k-1}(n+i d)\right)>$ $2 k>7$. When $(n, d, k)=(6,1,4)$, we check that (2) has no solution with $m \geq 2$.

Thus we may now assume $d=1$ and $n \leq k$. Recall that $k \geq 4$. For $n+k \leq 11$, we check that conditions of Lemma 4.1 are satisfied except when $n+k=11$. For $n+k=11$, we check that equation (2) has no solution with $m \geq 2$. Therefore we now assume that $n+k \geq 12$. By Lemma 2.8 , the interval $[2(n+k-1) / 3, n+k-1]$ contains a prime. Since $n \leq k$, we have $2(n+k-1) / 3 \geq n$ and consequently

$Q=P(n(n+1) \cdots(n+k-1)) \geq \max \left\{\right.$ primes in $\left.\left[\frac{2(n+k-1)}{3}, n+k-1\right]\right\} \geq \frac{2(n+k-1)}{3}$.

Since $2 Q>n+k-1$, we deduce that there exists a unique integer $i$ in the interval $[0, k-1]$ such that $n+i=Q$. Further $Q \geq \frac{2(n+k-1)}{3}>\frac{k+1}{2}$. Hence by Lemma 4.1, we can assume that $Q=7$. Then $7=Q \geq 2(n+k-1) / 3 \geq \frac{22}{3}>7$ since $n+k \geq 12$. This is a contradiction. Thus Theorem 2 is proved.

\section{Proof of Theorem 3}

We need the following main lemma for the proof of Theorem 3.

Lemma 5.1. Suppose there is an $i, 0 \leq i<k$ with $n+i d$ odd, $Q:=P(n+i d)>\frac{1+f(k, d)}{2}$ and $Q \nmid(n+j d)$ for $0 \leq j<k, j \neq i$. Then the equation (3) has no solution in positive integers $(n, d, k, b, y, m)$ with $P(b) \leq f(k, d)$.

Proof. Suppose the conditions of Lemma 5.1 are satisfied. Let $n+i d$ be odd and $Q:=P(n+i d)>\frac{1+f(k, d)}{2}$. Then $2 Q-1>f(k, d)$. Further write $n+i d=Q_{1} t$ where $Q_{1}=Q^{\nu}, \nu=\operatorname{ord}_{Q}(n+i d)$ and $\operatorname{gcd}(Q, t)=1$. Then $P(t)<Q$. From (3), we get

$$
v_{Q_{1}} \cdot \frac{v_{n+i d}}{v_{Q^{\nu}}} \prod_{j \neq i} v_{n+j d}=b y^{m}
$$

Let $p \mid v_{Q_{1}}$ and $p \neq 2$. By Corollary $2.2(i i)$, we have $p \geq 2 Q-1$. Since $2 Q-1 \geq Q>$ $p(t)$, we obtain from Corollary 2.4 that $\operatorname{gcd}\left(v_{Q_{1}}, \frac{v_{n+i d}}{v_{Q_{1}}}\right)=1$. Also for $0 \leq j<k, j \neq i$,

$$
\operatorname{gcd}\left(v_{Q_{1}}, v_{n+j d}\right)\left|\operatorname{gcd}\left(u_{2 Q_{1}}, u_{2(n+j d)}\right)=u_{\operatorname{gcd}\left(2 Q_{1}, 2(n+j d)\right)}\right| u_{2}=2
$$

since $Q \nmid(n+j d)$. Hence $\operatorname{gcd}\left(v_{Q_{1}}, v_{n+j d}\right)=2$. Now (16) together with $P(b) \leq$ $f(k, d)<2 Q-1 \leq p$ for every odd prime divisor of $v_{Q_{1}}$ implies $v_{Q_{1}}=2 y_{1}^{m}$ for some positive integer $y_{1}$. By Lemma 2.5, we get $Q_{1}=1$ which is not possible since $Q_{1} \geq Q>1$. Hence the assertion.

Proof of Theorem 3: The case $k=1$ follows by Lemma 2.5. Hence we consider $k \geq 2$. Let $k=2$. Then we have $v_{n} v_{n+d}=b y^{m}$ with $P(b) \leq 3$. By Lemma $2.3(d)$, we get either $v_{n}=2 y_{1}^{m}, v_{n+d}=b^{\prime} y_{2}^{m}$ or $v_{n}=b^{\prime} y_{1}^{m}, v_{n+d}=2 y_{2}^{m}$ with $P\left(b^{\prime}\right) \leq 3$. By 
Lemma 2.5, we get $v_{n}=v_{1}=2$ in the first case and $v_{n+d}=v_{1}=2$ in the latter case. The latter case is not possible and we have $n=1, v_{1} v_{1+d}=2 v_{1+d}=b^{\prime} y^{m}$ for some $b^{\prime}$ with $P\left(b^{\prime}\right) \leq 3$. If $3 \nmid b^{\prime}$, we get from Lemma $2.3(a)$ that $v_{1+d}=2 y^{m}$ which is again not possible by Lemma 2.5. Thus $3 \mid b^{\prime}$ and we have $v_{1+d}=2 \cdot 3^{r} y^{m}$ for some $r>0$. By Lemma $2.3(b)$, we get $1+d \equiv 2$ modulo 4 or $d \equiv 1$ modulo 4 . This is the case $k=2$ of Theorem 3 .

Let $k=3$. We need to solve $v_{n} v_{n+d} v_{n+2 d}=b y^{m}$ with $P(b) \leq 5$. By Lemma $2.3(c)$, we obtain $P(b) \leq 3$. By Lemma $2.3(d)$, we get $v_{n} v_{n+d}=b_{1} y_{1}^{m}$ and $v_{n+d} v_{n+2 d}=b_{2} y_{2}^{m}$ for some $b_{1}, b_{2}$ with $P\left(b_{1} b_{2}\right) \leq 3$. By the case $k=2$, we get $n+d=1$ from the latter equation which is not possible.

Let $k=4$ and we consider the equation $v_{n} v_{n+d} v_{n+2 d} v_{n+3 d}=b y^{m}$ with $P(b) \leq 7$. By Lemma $2.3(d)$, we observe that either $7\left|v_{n}, 7\right| v_{n+3 d}$ or 7 divides at most one of $v_{n}, v_{n+d}, v_{n+2 d}, v_{n+3 d}$. Again by Lemma $2.3(b)$ and $(c)$, there is at most one $v_{n+i d}, 0 \leq$ $i<4$ which is divisible by 3 since $\operatorname{gcd}(n, d)=1$ and also none of them is divisible by 5 . Therefore at least one $i \in\{1,2,3\}$ such that $\operatorname{gcd}\left(v_{n+i d}, 3 \cdot 5 \cdot 7\right)=1$. Hence from the equation (3), by using Lemma $2.3(d)$, we get $v_{n+i d}=2 y_{1}^{m}$ for some $y_{1}$. This is not possible by Lemma 2.5.

The case $k=5$ follows from case $k=4$ since $P(b) \leq 10$ is same as $P(b) \leq 7$. Thus $k \geq 6$. Let $d>1$. Observe that a prime $p>k$ divides at most one of $n+i d$ with $0 \leq i<k$ and $2 p \geq 2(k+1)>1+2 k$. Hence the assertion of Theorem 3 follows from Lemma 5.1 and Theorem 5 .

Therefore $d=1$. For $n+k \leq 11$, we check that (3) has no solution. Hence we suppose that $n+k \geq 12$. Let $n \leq 2 k$. Then $\frac{2}{3}(n+k-1) \geq n$ if $n<2 k-1$ and $\left\lceil\frac{2}{3}(n+k-1)\right\rceil=n$ if $n \in\{2 k-1,2 k\}$. By Lemma 2.8 and $n+k \geq 12$, the interval $\left(\frac{2}{3}(n+k-1), n+k-1\right]$ contains a prime and let $Q$ be the largest prime in the interval. Then $Q=n+i$ for some $i, 0 \leq i<k$ and $n+i$ is odd. Further $Q>\frac{2}{3}(n+k-1)$ implying $2 Q>\frac{4}{3}(n+k-1)>k$ so that $Q \nmid(n+j)$ for $0 \leq j<k, j \neq i$. Also

$$
2 Q-1 \geq \frac{4}{3}(n+k-1)-1 \geq \begin{cases}\frac{4 k}{3}-1>k & \text { if } n \leq k \\ \frac{8 k}{3}-1>2 k & \text { if } n>k\end{cases}
$$

since $k \geq 6$ implying $2 Q-1>f(k, d)$. By Lemma 5.1, assertion of Theorem 3 follows.

Thus $n>2 k$. Then the odd terms of among $\{n, n+d, \ldots, n+(k-1) d\}$ are given by

$$
\begin{array}{r}
n, n+2, \ldots, n+2\left[\frac{k-1}{2}\right] \text { if } n \text { is odd } \\
n+1, n+3, \ldots, n+1+2\left[\frac{k-2}{2}\right] \text { if } n \text { is even }
\end{array}
$$


and hence there are at least $1+\left[\frac{k-2}{2}\right]=\left[\frac{k}{2}\right]$ consecutive odd terms. Since $k \geq 6,\left[\frac{k}{2}\right] \geq$ 3. Let $S=\{(5,2),(7,2),(25,2),(243,2),(9,4),(13,5),(17,6),(15,7),(21,8),(19,9)\}$. For $\left(n,\left[\frac{k-1}{2}\right]+1\right) \in S$ if $n$ is odd and $\left(n+1,\left[\frac{k}{2}\right]\right) \in S$ if $n$ is even, we check that conditions of Lemma 5.1 are valid and hence (3) has no solution in this case. Thus we now suppose that $\left(n,\left[\frac{k-1}{2}\right]+1\right) \notin S$ if $n$ is odd and $\left(n+1,\left[\frac{k}{2}\right]\right) \notin S$ if $n$ is even. Then by Lemma 2.11, the greatest prime factor of these consecutive odd terms is at least $3.5\left[\frac{k}{2}\right] \geq 3.5\left(\frac{k-1}{2}\right)>k$ and hence there is an odd term $n+i$ for which $P(n+i)>k$. Since $P(b) \leq 2 k$, the assertion of Theorem 3 follows from Lemma 5.1.

\section{REFERENCES}

[1] M. A. Bennett and C. M. Skinner, Ternary Diophantine Equations via Galois Representations and Modular Forms, Canad. J. Math, 56 (2004), 23-54.

[2] N. Bicknell, A primer on the Pell sequence and related sequence, Fibonacci Quart., 13 (4) (1975), 345-349.

[3] Y. Bugeaud, M. Mignotte, and S. Siksek, Classical and modular approaches to exponential Diophantine equations. I. Fibonacci and Lucas perfect powers, Ann. of Math. 163 (2006), 969-1018.

[4] J. H. E. Cohn, Perfect Pell powers, Glasgow Math. J. 38 (1996), 19-20. MR97b:11047.

[5] P. Erdős and J. L. Selfridge, The product of consecutive integers is never a power, Illinois J. Math. 19 (1975) 292-301.

[6] A. F. Horadam, Applications of Modified Pell Numbers to Representations, Ulam Quart., 3 (1994), 34-53.

[7] E. Kilic and D. Tasci, The Linear Algebra of The Pell Matrix, Bol. Soc. Mat. Mexicana, 3 (11) (2005).

[8] T. Koshy, Fibonacci and Lucas Numbers with Applications, Wiley-Interscience Publications, 2001.

[9] F. Luca and T. N. Shorey, Diophantine equations with products of consecutive terms in Lucas sequences, J. Number Theory, 114 (2005), 298-311.

[10] T. Nagell, Sur une classe d'équations exponentielles, Ark. Mat. 3 (1958), 569-582.

[11] A. Pethő, Perfect powers in second order linear recurrences, J. Number Theory 15 (1982) $5-13$.

[12] A. Pethő, The Pell sequence contains only trivial perfect powers. Sets, graphs and numbers (Budapest, 1991), 561-568, Colloq. Math. Soc. János Bolyai, 60, North-Holland, Amsterdam, 1992. MR94e:11031.

[13] J. B. Rosser and L. Schoenfeld, Approximate formulas for some functions of prime numbers, Illinois J. Math., 6 (1962), 64-94.

[14] S. Laishram and T. N. Shorey, Number of prime divisors in a product of terms of an arithmetic progression, Indag. Math., 15(4), (2004), 505-521.

[15] Shanta Laishram and T. N. Shorey, The greatest prime divisor of a product of consecutive integers, Acta Arith. , 120 (2005), 299-306.

[16] Shanta Laishram and T. N. Shorey, The greatest prime divisor of a product of terms in an arithmetic progression, Indag. Math. , 17(3) (2006), 425-436.

[17] Shanta Laishram and T. N. Shorey, Irreducibility of generalized Hermite-Laguerre polynomials, Funct. Approx. Comment. Math., 47 (2012), part 1, 51-64.

[18] N. Saradha, T. N. Shorey and R. Tijdeman, Some extensions and refinements of a theorem of Sylvester, Acta Arith. 102 (2002), 167-181. 
[19] T. N. Shorey and C. L. Stewart, On the Diophantine equation $a x^{2 t}+b x^{t} y+c y^{2}=d$ and pure powers in recurrence sequences, Math. Scand. 52 (1983) 24-36.

[20] T. N. Shorey and R. Tijdeman, On the greatest prime factor of an arithmetical progression, A tribute to Paul Erdős, ed. by A. Baker, B. Bollobás and A. Hajnal, Cambridge University Press (1990), 385-389.

[21] T. N. Shorey, Exponential Diophantine equations involving products of consecutive integers and related equations, in: R. P. Bambah, V.C. Dumir, R. J. Hans-Gill (Eds.), Number Theory, Hindustan Book Agency, 1999, pp. 463-495.

[22] J. J. Sylvester, On arithmetical series, Messenger of Mathematics, XXI (1892), 1-19, 87-120, and Mathematical Papers, 4 (1912), 687-731.

[23] B. M. M. de Weger, Algorithms for Diophantine equations, CWI Tract, 65. Stichting Mathematisch Centrum, Centrum voor Wiskunde en Informatica, Amsterdam, 1989.

Departamento de Matemáticas, Universidad del Cauca, Calle 5 No 4-70, Popayán, Colombia.

E-mail address: jbravo@unicauca.edu.co

Stat-Math Unit, India Statistical Institute, 7, S. J. S. Sansanwal Marg, New Delhi, 110016, INDIA

E-mail address: pranabesh.math@gmail.com

Centro de Ciencias Matemáticas, Universidad nacional Autónoma de México, Ap. Postal 61-3 (Xangari), C.P. 58089, Morelia, Michoacán, Mexico.

E-mail address: sguzman@matmor.unam.mx

Stat-Math Unit, India Statistical Institute, 7, S. J. S. Sansanwal Marg, New Delhi, 110016, INDIA

E-mail address: shanta@isid.ac.in 